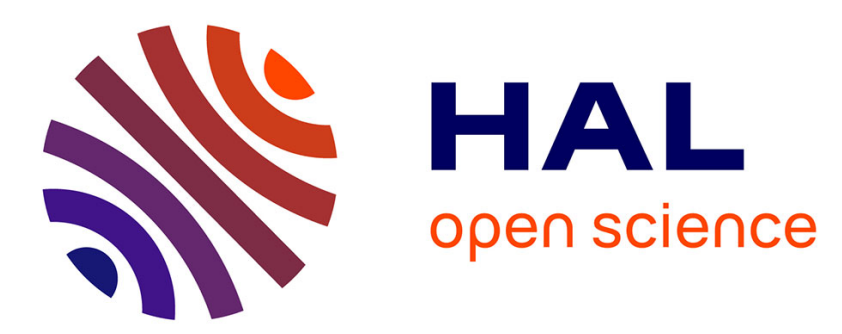

\title{
Dynamical Coupling between Connected Foam Films: Interface Transfer across the Menisci
}

Adrien Bussonnière, Evgenia Shabalina, Xavier Ah-Thon, Mickaël Le Fur, Isabelle Cantat

\section{- To cite this version:}

Adrien Bussonnière, Evgenia Shabalina, Xavier Ah-Thon, Mickaël Le Fur, Isabelle Cantat. Dynamical Coupling between Connected Foam Films: Interface Transfer across the Menisci. Physical Review Letters, 2020, 124 (1), pp.018001. 10.1103/PhysRevLett.124.018001 . hal-02469672

\section{HAL Id: hal-02469672}

\section{https://hal-univ-rennes1.archives-ouvertes.fr/hal-02469672}

Submitted on 6 Feb 2020

HAL is a multi-disciplinary open access archive for the deposit and dissemination of scientific research documents, whether they are published or not. The documents may come from teaching and research institutions in France or abroad, or from public or private research centers.
L'archive ouverte pluridisciplinaire HAL, est destinée au dépôt et à la diffusion de documents scientifiques de niveau recherche, publiés ou non, émanant des établissements d'enseignement et de recherche français ou étrangers, des laboratoires publics ou privés. 


\title{
Dynamical Coupling between Connected Foam Films: Interface Transfer across the Menisci
}

\author{
Adrien Bussonnière, Evgenia Shabalina, Xavier Ah-Thon, Mickaël Le Fur, and Isabelle Cantat \\ Univ Rennes, CNRS, IPR (Institut de Physique de Rennes)-UMR 6251, F-35000 Rennes, France
}

(Received 31 January 2019; revised manuscript received 10 September 2019; published 3 January 2020)

\begin{abstract}
The highly confined flow of the liquid phase, trapped between the gas bubbles, is at the origin of the large effective viscosity of the liquid foams. Despite the industrial relevance of this complex fluid, the foam viscosity remains difficult to predict because of the lack of flow characterization at the bubble scale. Using an original deformable frame, we provide the first experimental evidence of the interface transfer between a compressed film (or a stretched film) and its first neighbor, across their common meniscus. We measure this transfer velocity, which is a key boundary condition for local flows in foams. We also show the dramatic film thickness variation induced by this interface transfer, which may play an important role in the film thickness distribution of a 3D foam sample.
\end{abstract}

DOI: 10.1103/PhysRevLett.124.018001

Dry liquid foams behave as shear thinning complex fluids, having a surprisingly high effective viscosity [1]. The macroscopic deformations turn at local scale into bubble deformations and reorganizations. Foam coarsening or collapse induce similar local motions. The timescales of these dissipative deformations are at the origin of the effective foam viscosity and are governed by the fundamental dissipative coefficients of the system which are (i) the bulk viscosity, involved in case of thin film extension, velocity difference between both film interfaces, or volume exchange between films and menisci; (ii) the interface shear viscosity, involved in case of in-plane film shear; and (iii) the diffusion coefficients and absorption or desorption timescales, important when the effective foam viscosity is induced by the surface tension variations and thus by the surfactant transport [2]. Surfactant convection implies that case (iii), as cases (i) and (ii), involves the velocity field in the liquid phase. The determination of the relative importance of these various dynamical processes remains an open problem, which motivated numerous experimental and theoretical studies at the scale of few foam films. In these studies, the film deformations are imposed either by moving the system boundaries [3-8] or by forcing $T 1$ transformations [9-16], and, in both cases, the models used to rationalized the observations rely on a strong assumption on a precise quantity: the surfactant surfacic flux $j_{s}=\Gamma v_{s}$ across a meniscus, from one film to its neighbor, with $\Gamma$ the surfactant interfacial concentration and $v_{s}$ the interfacial velocity at the meniscus. A generic situation is shown in Fig. 1, corresponding to the case addressed in [9].

This crucial parameter $j_{s}$ controls the coupling between one film to another in all kinds of foam deformation involving film area variations. As the variations of surface tension of deformed foam films are relatively small (a few $\mathrm{mN} / \mathrm{m}$ ), the surface concentration $\Gamma$ remains close to its equilibrium value $\Gamma_{\mathrm{eq}}$ and the flux $\Gamma v_{s}$ is mainly governed by the interfacial velocity. In the literature, several phenomenological rules are proposed: (i) the full transfer, proposed in [9] and imposing $v_{s}=d L / d t$, with $d L / d t$ the film length variation defined in Fig. 1; (ii) the absence of flux with $v_{s}=0$ [12]; or (iii) an ad hoc law, between the two previous limiting cases $[3,13]$.

This Letter provides the first experimental determination of $v_{s}$, on both sides of a meniscus. We show the following. (i) For the chosen deformation and foaming solution, $v_{s}$ has comparable values on both sides of the menisci. Identifying $j_{s}$ with $\Gamma_{\mathrm{eq}} v_{s}$, this indicates that the meniscus does not play the role of a perfect surfactant reservoir. (ii) The obtained value for $v_{s}$ is smaller than the film length time derivative, but of the same order. These results show evidence of the important coupling between adjacent foam films and the feasibility and the need of a systematic measure of $j_{s}$,

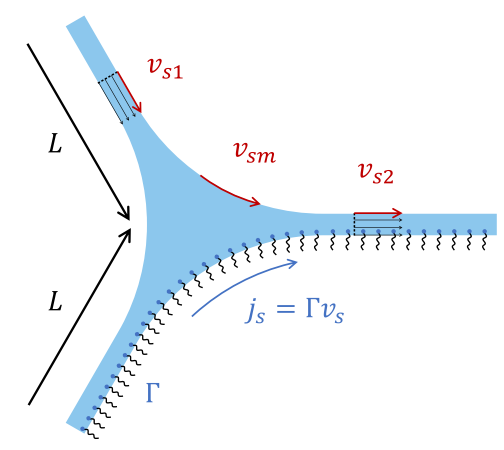

FIG. 1. Scheme of the interfacial flow in the vicinity of a meniscus, driven, for example, by the decrease of the size $L$ of the lateral films. 


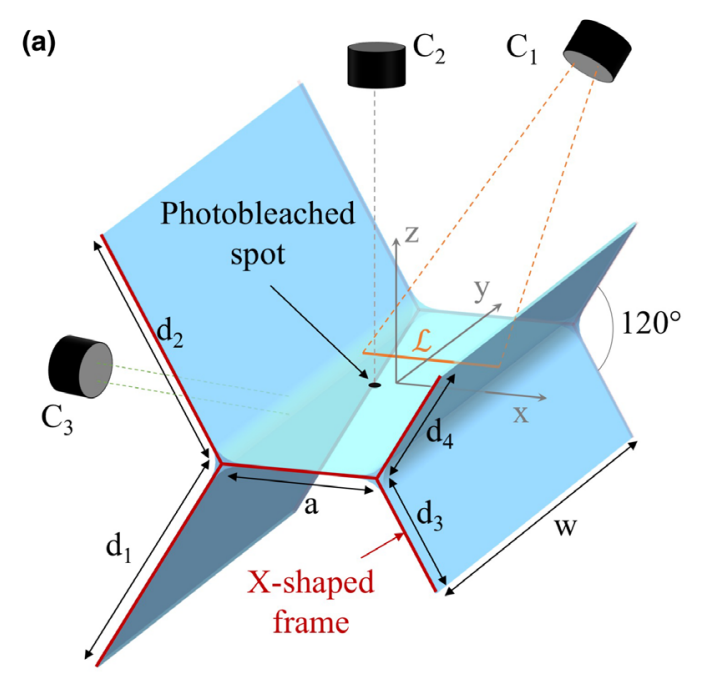

(b)
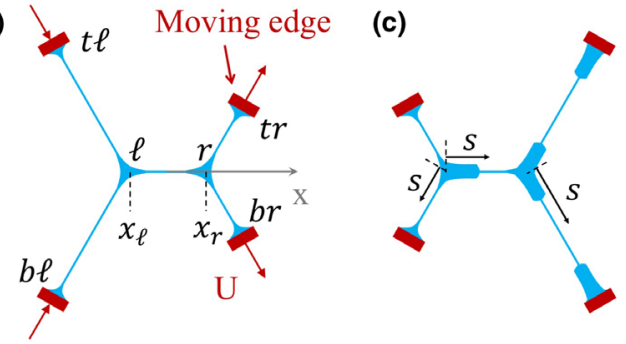

FIG. 2. (a) Scheme of the setup. The five foam films and the two free menisci are represented (in blue), as well as the front part of the solid frame ( $X$-shaped piece, in red). The dimensions are $a=6 \mathrm{~mm}, w=41.5 \mathrm{~mm}$. (b) Representation of the system in the $(x, z)$ plane, at the time $t=0$. The four moving edges (top and bottom left $t \ell, b \ell$, and top and bottom right $t r, b r)$ are shown in red. (c) The same system, after motor motion. The thick parts of the central and right films are the pieces of film extracted from the meniscus.

which appears as an unavoidable preliminary step to improve local models of foam rheology.

We design a dedicated frame, shown in Fig. 2(a), on which a horizontal central film is connected to four lateral films, labeled by the index $i \in[1 ; 4]$, whose lengths are controlled by linear piezomotors. This geometry has been chosen so that each film remains flat and stays in the same plane during the deformation.

The lateral films make an angle of $120^{\circ}$ with the central film, as prescribed by the two $X$-shaped metallic pieces constituting the lateral boundaries of the system [see Fig. 2(a)]. We impose a deformation between an initial shape and its mirror image [see Figs. 2(b) and 2(c)]: the distance $d_{i}(t)$ between the moving external edge $i$ and the corresponding meniscus is $d_{1,2}=d_{\ell}=d-U t$ on the left side and $d_{3,4}=d_{r}=d+U(t-\tau)$ on the right side, between $t=0$ and $t=\tau$. The four motors stop after a displacement of $10 \mathrm{~mm}$, at time $\tau=0.2 \mathrm{~s}$. The motor velocity is $U=50 \mathrm{~mm} / \mathrm{s}$ and $d=17.1 \mathrm{~mm}$. Syringes positioned along the four upper arms of the $X$-shaped pieces each inject a flux $Q=0.05 \mathrm{~mL} / \mathrm{min}$ of surfactant solution into the system. This allows us to prepare a reproducible initial state for the films and menisci.

The solution is made of deionized water, sodium dodecyl sulfate $(\mathrm{SDS}, \mathrm{cmc}=2.36 \mathrm{~g} / \mathrm{L}$ ) at $5.6 \mathrm{~g} / \mathrm{L}$, dodecanol at $0.05 \mathrm{~g} / \mathrm{L}$, glycerol at $15 \%$ in volume, and fluorescein at $0.8 \mathrm{~g} / \mathrm{L}$. The viscosity is $\eta=1.52 \mathrm{mPa}$ s, and the equilibrium surface tension is $\gamma=32 \mathrm{mN} / \mathrm{m}$.

The dynamical behavior is fully characterized by simultaneously measuring the film thickness and velocity. The film thickness $h$ is measured on a line $\mathcal{L}$ oriented perpendicular to the free menisci [see Fig. 2(a)], with a spectral camera $\left(C_{1}\right)$ Resonon Pika L (see Supplemental Material A [17]) illuminated with a halogen light. The velocity field is deduced by the motion of photobleached spot, monitored with the camera $C_{2}$. A $488 \mathrm{~nm}$ laser is split in two beams: one is expanded to illuminate a large part of the film; and the other is focused during $15 \mathrm{~ms}$, prior to the motor motion, to locally photobleach the fluorescein (see [18] for more details). This technique is well suited to probe soap film motion since the velocity is uniform over the film thickness in flat film due to (i) the uniformity of the pressure preventing any Poiseuille flow and to (ii) the symmetry of the system and of the imposed deformations implying identical interface velocities and no shear (far enough from the menisci, see Fig. 1). Moreover, the experimental time is shorter than the fluorescein diffusion time. Consequently, the spot remains well contrasted along its whole trajectory and is a good passive tracer.

The camera $C_{2}$ is also used to track the positions $x_{\ell}(t)$ and $x_{r}(t)$ of the left and right menisci, respectively. They slightly move during the deformation, without modifying significantly the central film area. In the central film, we denote by $s$ the distance to the left meniscus, i.e., $s=x-x_{\ell}(t)$, while in the lateral films $s$ is the distance to the corresponding free meniscus [see Fig. 2(c)].

Finally, the vertical dimension $w_{m}$ of the left meniscus is measured before deformation by light transmission using a collimated white light and a telecentric objective Edmund Optics Silver-TL $4 \times$ (camera $C_{3}$ ).

The thickness profiles during and after motor motion are shown in Fig. 3 for different times, in the central, left, and right films, as well as the volumes (per unit length in the $y$ direction). These plots show evidence of the following phenomenology.

(i) Initial state $(t<0)$. - The central film thickness is uniform and equal to $h_{0}=400 \pm 200 \mathrm{~nm}$. The left film is $3 \mu \mathrm{m}$ thick and the right film is $1.5 \mu \mathrm{m}$ thick. These last thicknesses have steady but out of equilibrium values, which depend on the film size and inclination, and on the injected flux. The meniscus radius of curvature is deduced from the equilibrium relationship $r_{m}=w_{m}=320 \mu \mathrm{m}$ [1].

(ii) Lateral films stretching and compression $(t \in[0-0.1] \mathrm{s})$.- - The right film gets thinner and the left film gets thicker, but their volumes remains constant. This shows evidence of a homogeneous film deformation with 

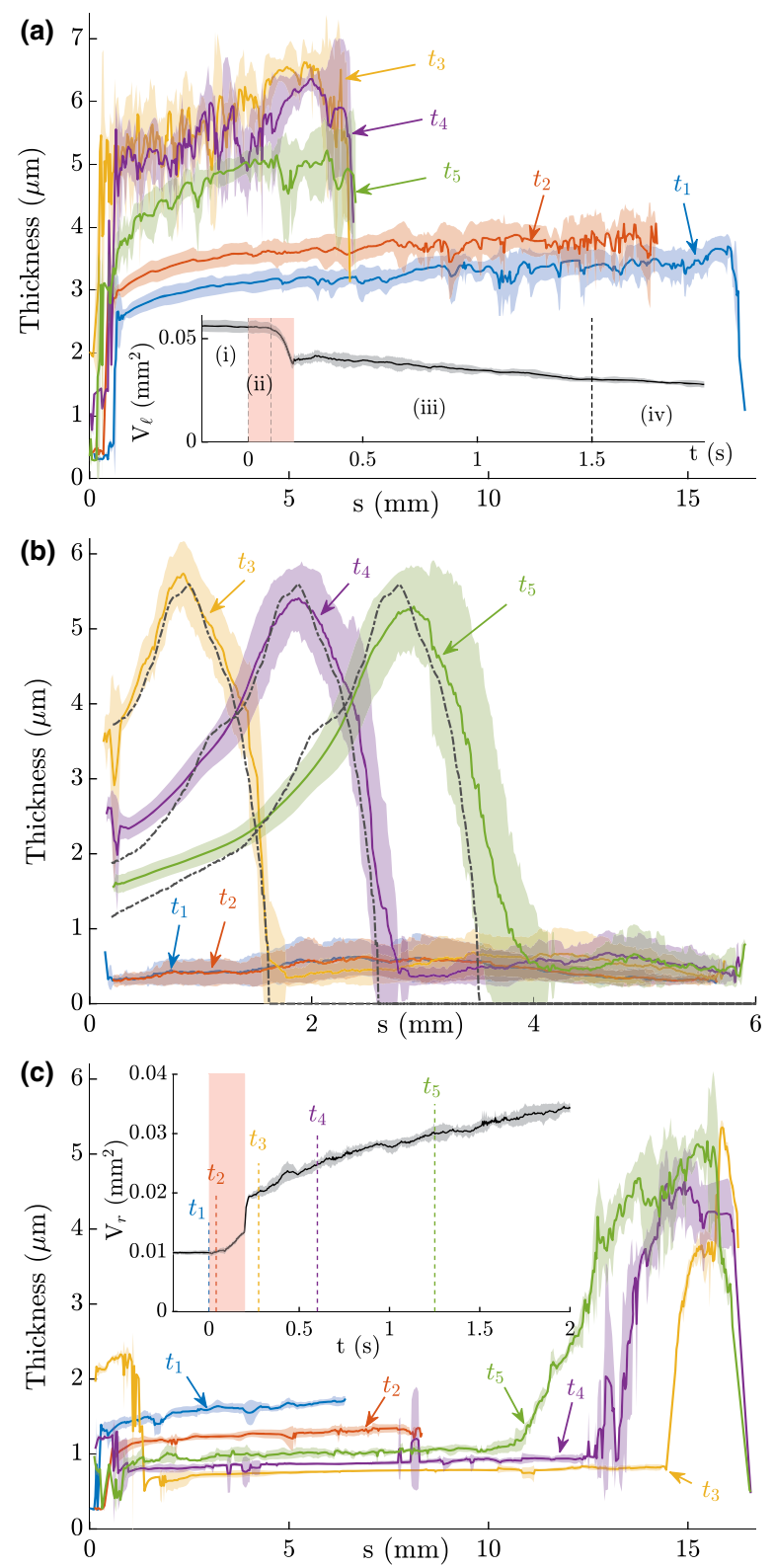

FIG. 3. Film thickness as a function of $s$ [defined in Fig. 2(c)], in the bottom left film (a), the central film (b), and the bottom right film (c), at times $t_{1}=0 \mathrm{~s}$ [step (i)]; $t_{2}=0.04 \mathrm{~s}$ [step (ii)]; $t_{3}=$ $0.075 \mathrm{~s}$ [step (iii), before the gravitational instability], and $t_{4,5}=$ $[0.6,1.25] \mathrm{s}$ [step (iii), after instability]. The solid lines represent the average of five experiments for the lateral films and 50 for the central, the shaded areas are the standard deviation. The dashed lines in (b) correspond to the thickness profiles predicted by Eq. (2). The insets show the left (a) and right (c) film volumes (per unit length in the $y$ direction) $V_{\ell}$ and $V_{r}$, and indicate the times $t_{i}$, time domains (i)-(iv), and motor motion time (shaded red).

no exchange with the menisci. No noticeable evolution is observed in the central film.

(iii) Interface transfer across menisci $(t \in[0.1-1.5] \mathrm{s})$.In the right film, thick pieces of film are extracted from both the right and bottom right menisci [see Fig. 2(c)]. A straight, well-defined, frontier separates these extracted films from the film initially present (hereafter called the initial film). These steps (ii) and (iii) were already observed in a stretched film in [5] and in [15]. Similarly, in the compressed film (left), the film volume decreases, showing evidence of a liquid flux toward the menisci [see Fig. 3(a), inset]. In the central film, a thick film is extracted from the left meniscus and the thin film initially present flows into the right meniscus. This motion happens without any deformation of the film, for which size and position remain constant, and is thus solely induced by the interface transfer from the adjacent films.

(iv) Eventually, the system recovers its slow drainage behavior, after a delay of the order of $10 \mathrm{~s}$.

Note that the top-bottom mirror symmetry of the motion in the right film is broken at $t \approx 0.25 \mathrm{~s}$ by a gravitational instability which imposes a stratification of the film thickness, thicker parts of the film laying below the thinner parts. A single extracted film is thus observed at later times in the right film, close to the bottom meniscus. Some film is, however, continuously extracted at the top, but it falls down after its extraction from the meniscus and merges with the thick film produced at the bottom (see [19]).

The motion of the central film is quantified from the velocity measurements. We measured the photobleached spots position relatively to the left meniscus $s(t)=x(t)-$ $x_{\ell}(t)$. The displacement of the spots in the $y$ direction remains smaller than $10 \%$ of the total spot displacement, except for positions very close from the right meniscus, where we observe marginal regeneration which locally breaks the invariance in the $y$ direction [20]. In the following, the spots at a distance smaller than $1 \mathrm{~mm}$ from the right meniscus are discarded. We recorded four trajectories $s_{i}(t)$, each being averaged over ten experiments. The index $i \in[1-4]$ refers to the initial distance $s_{i}(0)$ between the spots (i) and the left meniscus, ranging from 1 to $4 \mathrm{~mm}$.

These trajectories show evidence of a translational motion of the central film, with almost no deformation, over a distance which is a significant fraction of the motor displacement. Figure 4 (inset) shows the distance between the spots [(2)-(4)] and the spots (1) as a function of time. The spots (1) and (2) are close to the left meniscus, and the distance between them is almost conserved during the whole motion, showing the absence of film deformation in this region. The distances between spots (3), (4) and spot (1) slightly decrease, indicating a small film compression in the right part of the film. However, no clear signature of this compression is observable in the thickness map [Fig. 3(b)], and residual recirculations close to the right meniscus may be at the origin of this small drift, especially at the longest times.

The trajectory of spots (1) relatively to their initial position, i.e., the distance $L^{c}(t)=s_{1}(t)-s_{1}(0)$, is plotted in Fig. 4. As the central film moves as a whole close to the left meniscus, $L^{c}$ corresponds to the amount of film extracted from the left meniscus. If we neglect the small 




FIG. 4. Amount of interface $L$ transferred from the compressed side to the stretched side as a function of time. Black, full line: $L_{c}(t)$ (central film); red, full line: $L_{\text {sym }}^{\ell}$; dashed line: $L^{\ell}$ (compressed film); blue: $L_{\text {sym }}^{r}$ (stretched film). The dashed black line shows the motor displacement, stopping at the dotted black line. Inset: trajectories of the spots (2-4, colored) and of the frontier of the extracted film (black) relatively to the spots (1). Error bars are the standard deviation for ten trajectories.

compression observed close to the right meniscus, it corresponds also to the amount of film absorbed by the right meniscus.

We now compare this transferred length $L^{c}(t)$ to the amount of interface $L^{\ell}(t)$ going from the compressed film to the left meniscus, and to the amount $L^{r}(t)$ going from the right meniscus to the stretched film.

As the direct velocity measurements from the photobleached trajectories are not feasible because of the gravitational instabilities, these quantities are determined from the thickness profile only, at the price of an additional assumption: we assume that the same amount of interface goes in (or out) the top and bottom meniscus bounding the lateral film. In that case, we establish in Supplemental Material B [17] that

$$
v_{r, \ell}^{\mathrm{sym}}(0, t)=\frac{1}{2}\left(\dot{d}_{r, \ell}-d_{r, \ell} \frac{\varepsilon_{r, \ell}}{\varepsilon_{r, \ell}}\right),
$$

with $v_{r, \ell}^{\text {sym }}(0, t)$ the film velocity at the free meniscus and $\varepsilon_{r, \ell}=\left\langle h_{r, \ell}\right\rangle_{(t=0)} /\left\langle h_{r, \ell}\right\rangle_{(t)}$ the film extension in the right and left films, respectively. The spatial average \langle\rangle is made, respectively, on the whole left film, and on the thin part of the right film. Then we define $L_{\mathrm{sym}}^{r}(t)=\int_{0}^{t} v_{r}^{\mathrm{sym}}(0, t) d t$ and $L_{\mathrm{sym}}^{\ell}(t)=-\int_{0}^{t} v_{\ell}^{\mathrm{sym}}(0, t) d t$.

In the right film, the top and bottom extracted films have the same width before the instability begins, thus confirming the validity of our symmetry assumption, at least at short time. In the left film, a spot trajectory $s_{\alpha}(t)$ has been obtained, again at short time, allowing us to compute $v_{\ell}$ as $v_{\ell}(0, t)=\dot{s}_{\alpha}(-\dot{\varepsilon} / \varepsilon) s_{\alpha}$, which does not require any symmetry assumption (see Supplemental Material B [17]).
The prediction $L^{\ell}(t)=-\int_{0}^{t} v_{\ell}(0, t) d t$ is in qualitative agreement with $L_{\text {sym }}^{\ell}(t)$, as shown in Fig. 4.

The quantities $L_{\text {sym }}^{r}$ and $L_{\text {sym }}^{\ell}$ show a behavior very similar to $L^{c}$ with time (Fig. 4). A first conclusion is thus that the motor displacements lead to the transfer of the interface from the left film to the right film across the two free menisci and the central film, occurring with negligible in-plane deformation, and with some delay with respect to the motor motion. The amount of interface transferred after $1 \mathrm{~s}$ is of the order of $3 \mathrm{~mm}$, which is a significant part of the $10 \mathrm{~mm}$ displacement of the motor.

This interface transfer across the central film is coupled to the extraction of thick film from the left meniscus. It is separated from the thin film by a sharp frontier, moving at the velocity $v_{c}(t)=d L^{c} / d t$ (see Fig. 4 inset).

The thickness of the film extracted from a meniscus of radius $r_{m}$, at a constant velocity $v$ is given by the Frankel's expression $h^{\mathrm{Fr}}=2.66 r_{m}(\eta v / \gamma)^{2 / 3}$ [20]. In the central film, the dynamical meniscus lateral extension scales as $\delta_{\text {dyn }}=$ $r_{m}\left(\eta v_{c} / \gamma\right)^{1 / 3} \sim 10 \mu \mathrm{m}$ and the film goes through this region in a time $t_{\mathrm{dyn}} \sim \delta_{\mathrm{dyn}} / v_{c} \sim 10^{-3} \mathrm{~s}$. As $t_{\mathrm{dyn}} \ll \tau=$ $0.2 \mathrm{~s}$, the timescale of the velocity variations (see Fig. 4), an approximation of quasisteady motion is justified. The thickness gradient at the frontier is of the order of $\phi \approx$ $10^{-2}$ and it decreases on the typical timescale $\phi^{-4} \eta h / \gamma \approx$ $10 \mathrm{~s} \gg \tau$ [21]: once extracted from the meniscus, the film thus simply follows a passive convection. Moreover, as the central film extension rate is negligible, it moves without any deformation. As a consequence, the film at the position $s$ at time $t$ has been extracted from the meniscus at time $t_{0}(s)$ implicitly given by the relation $s=\int_{t_{0}(s)}^{t} v_{c}(t) d t$, and the theoretical prediction for its thickness is

$$
h^{\mathrm{th}}(s, t)=2.66 r_{m}\left(\frac{\eta v_{c}\left[t_{0}(s)\right]}{\gamma}\right)^{2 / 3} .
$$

This theoretical profile is plotted in Fig. 3(b) (dashed lines) and shows a quantitative agreement with the experimental data, which validates a posteriori the assumptions made.

In conclusion, we show evidence of and quantify interface transfer between adjacent foam films, which constitutes a first critical step toward more realistic foam models taking into account the coupling between films. Moreover, the imposed deformation induces a thickness increase by a factor of 10 in a film which is only a neighbor of the deformed films. The thickness relaxation toward its initial value lasts for several seconds. As a consequence, the localized deformations occurring in 3D foam samples, due to aging or global flow, may redistribute the liquid phase in the thin films at a much larger distance from the local deformation than previously expected. This process is potentially an efficient factor of rejuvenation of the foam films, competing with the slow drainage imposed by the meniscus capillary suction and gravity. This should be 
confronted to in situ film thickness measurements [22,23]. The original setup we developed proved its efficiency to identify deformation modes in foam films, and to measure them with an accuracy allowing for a quantitative comparison with local theoretical models. We believe this should remain the case for a large class of imposed deformations, which paves the way to a deeper understanding of dissipation in foams.

This project has received funding from the European Research Council (ERC) under the European Union's Horizon 2020 research and innovation program (Grant Agreement No. 725094). We thank A. Saint-Jalmes and A. Bérut for fruitful discussions and E. Schaub for technical support.

[1] I. Cantat et al., Foams. Structure and Dynamics (Oxford University Press, Oxford, 2013).

[2] D. Buzza, C.-Y. Lu, and M. E. Cates, J. Phys. II (France) 5, 37 (1995).

[3] S. Besson and G. Debrégeas, Eur. Phys. J. E 24, 109 (2007).

[4] S. Besson, G. Debrégeas, S. Cohen-Addad, and R. Höhler, Phys. Rev. Lett. 101, 214504 (2008).

[5] J. Seiwert, M. Monloubou, B. Dollet, and I. Cantat, Phys. Rev. Lett. 111, 094501 (2013).

[6] S. Costa, R. Höhler, and S. Cohen-Addad, Soft Matter 9, 1100 (2013).

[7] S. Costa, S. Cohen-Addad, A. Salonen, and R. Höhler, Soft Matter 9, 886 (2013).

[8] A. Titta, M. Le Merrer, F. Detcheverry, P. Spelt, and A.-L. Biance, J. Fluid Mech. 838, 222 (2018).
[9] M. Durand and H. A. Stone, Phys. Rev. Lett. 97, 226101 (2006).

[10] A. L. Biance, S. Cohen-Addad, and R. Höhler, Soft Matter 5, 4672 (2009).

[11] N. D. Denkov, S. Tcholakova, K. Golemanov, and A. Lips, Phys. Rev. Lett. 103, 118302 (2009).

[12] P. Grassia, B. Embley, and C. Oguey, J. Rheol. 56, 501 (2012).

[13] R. Satomi, P. Grassia, and C. Oguey, Colloids Surf. A 438, 77 (2013).

[14] P. Petit, Ph.D. thesis, Université Claude Bernard-Lyon I, 2014.

[15] P. Petit, J. Seiwert, I. Cantat, and A.-L. Biance, J. Fluid Mech. 763, 286 (2015).

[16] F. Zaccagnino, A. Audebert, and S. J. Cox, Phys. Rev. E 98, 022801 (2018).

[17] See Supplemental Material at http://link.aps.org/ supplemental/10.1103/PhysRevLett.124.018001 for information about the spectral camera and the calculation of the transmitted interface length.

[18] J. Seiwert, R. Kervil, S. Nou, and I. Cantat, Phys. Rev. Lett. 118, 048001 (2017).

[19] E. Shabalina, A. Bérut, M. Cavelier, A. Saint-Jalmes, and I. Cantat, Phys. Rev. Fluids 4, 124001 (2019).

[20] K. J. Mysels, K. Shinoda, and S. Frankel, Soap Films: Study of their Thinning and a Bibliography (Pergamon, NewYork, 1959).

[21] Y. Chai, T. Salez, J. D. McGraw, M. Benzaquen, K. Dalnoki-Veress, E. Raphael, and J. A. Forrest, Science 343, 994 (2014).

[22] M. Axelos and F. Boué, Langmuir 19, 6598 (2003).

[23] C. Micheau, P. Bauduin, O. Diat, and S. Faure, Langmuir 29, 8472 (2013). 